\title{
Article \\ Numerical Study of the Emission of Acoustic Energy of Single Collapsing Vapor Bubble near a Rigid Wall
}

\author{
Hangbo Duan $(\mathbb{D}$, Linya Chen *(D) and Xiaoyu Liang *(D) \\ College of Metrology and Measurement Engineering, China Jiliang University, Hangzhou 310018, China; \\ s1902080409@cjlu.edu.cn \\ * Correspondence: chenlinya@cjlu.edu.cn (L.C.); xyliang@cjlu.edu.cn (X.L.)
}

\begin{abstract}
A compressible two-phase solver considering phase transition and thermodynamic effects is developed on OpenFOAM to numerically investigate the dynamics of single cavitation bubble collapse near the rigid wall. A grid independence analysis is conducted, and the accuracy of the pressure field is verified by comparing the numerical results with the experimental results. The emission process of acoustic energy during the bubble collapse is discussed. Moreover, the acoustic radiation energy in the flow field under different dimensionless bubble-wall distances $\gamma$ is further studied, which is related to the mechanism of cavitation erosion. The results show that $\gamma$-value has a significant effect on the amplitude of acoustic energy. The maximum amplitude of acoustic energy accounts for $8 \%$ to $25 \%$ of the total energy in the flow field when $\gamma$-value ranges from 0.1 to 2.0 . The amplitude of acoustic energy decreases with $\gamma$ in the range $0.1<\gamma<0.9$ while increases with $\gamma$ in the range $0.9<\gamma<2.0$.
\end{abstract}

Keywords: cavitation bubble; numerical simulation; acoustic energy; phase transition; compressibility

check for updates

Citation: Duan, H.; Chen, L.; Liang, $X$. Numerical Study of the Emission of Acoustic Energy of Single Collapsing Vapor Bubble near a Rigid Wall. Water 2022, 14, 455. https:// doi.org/10.3390/w14030455

Academic Editor: Francesco Napolitano

Received: 15 December 2021 Accepted: 29 January 2022

Published: 2 February 2022

Publisher's Note: MDPI stays neutral with regard to jurisdictional claims in published maps and institutional affiliations.

Copyright: () 2022 by the authors. Licensee MDPI, Basel, Switzerland. This article is an open access article distributed under the terms and conditions of the Creative Commons Attribution (CC BY) license (https:// creativecommons.org/licenses/by/ $4.0 /)$.

\section{Introduction}

Cavitation erosion is unavoidable in hydraulic machinery and equipment that operates for a long time [1-6], such as water turbines, pumps, and so forth. Therefore, it is crucial to assess the risk of erosion during the design stage of the equipment. When the pressure in the high-speed operation area of the equipment drops to a critical value-usually the saturated vapor pressure-cavitation bubbles nucleate and grow in this area, and then immediately collapse, passing the high-pressure area of flow [7,8]. During the bubble collapse phase in the vicinity of the material surfaces [9], high-speed micro-jets directed to the surfaces are generated, accompanied by the emission of shock waves, causing damage to the material. Generally, the erosion risk is assessed by experimental methods [10-12], for instance, utilizing a high-speed camera supplemented by paint testing to observe the operation of the prototype in the cavitation flow. These experimental methods are, however, expensive and used in the later stages of the design process. With the rapid development of computer capabilities in the past two decades, the idea of establishing a numerical prediction model to assess the risk of erosion has come true. Compared with the experimental methods, numerical methods are more competitive since they can predict the erosion intensity in the early design stage of equipment and reduce the cost greatly [13].

Despite extensive research, the mechanism of cavitation erosion is still controversial. Bark et al. [14] proposed a theoretical model and considered that the surface damage is mainly caused by the impact of micro-jets on the material surface. Based on this theory, Dular et al. [15] proposed a cavitation erosion model assuming that the surface erosion occurs when the velocity of the micro-jet exceeds a certain velocity threshold which is related to the characteristics of the material, thereby calculating the number of pits on the surface and the total loss area according to the velocity threshold. Following the approach of Dular, Peter et al. [16,17] developed a new cavitation model to predict the 
formation of micro-jets near surfaces, and further introduced dimensionless coefficients to enable a qualitative prediction of erosion, based on the amount of impacts and their intensity in a certain area. On the contrary, Fortes-Patella et al. [18-20] developed a different cavitation erosion model called energy balance approach based on the potential energy theory [21], considering that the erosion was caused by the shock wave emitted during the collapse phase impacting the material surface. The model analyzed the energy balance and conversion in the process from a large-scale vapor structure to the material surface based on the concept of energy level cascades. Sören et al. [22] also established the relationship between the volume change of the gas phase and the distribution of pressure energy received by the solid surface based on the potential energy theory, and developed a bubble collapse load model to predict the collapse load intensity under different cavitation flows. Besides, some scholars, for example, Arabnejad et al. [13] considered both micro-jets and shock-waves as the mechanisms for cavitation erosion, thereby presented a method to assess the risk of cavitation erosion based on the kinetic energy in the surrounding liquid during the collapse instead of the potential energy of collapsing cavities. We are more inclined to the energy balance approach since it is more applicable to the industrial pressure based unsteady Reynolds-averaged Navier-Stokes solver and is more conducive to the estimation of the cavitation erosion risk for industrial applications of hydraulic equipment [23].

Wall erosion is related to many factors, such as the distance between the wall and the cavitation bubble, the size of the bubble, the number of bubbles, and so forth. The mechanism exploration of impulsive pressure generation from a single bubble collapsing in the vicinity of a rigid wall is one of the most essential research areas concerned with cavitation erosion. Many experimental results show that the bubble-wall dimensionless distance $\gamma$ has a decisive influence on the wall impact pressure and cavitation erosion intensity, where $\gamma$ is defined as the ratio of the distance between the wall and the initial bubble center $L$ to the maximum radius of the bubble $R_{0}$. Philipp et al. [24] examined the dynamics of single cavitation bubble under different $\gamma$ conditions in detail, and found that the wall erosion occurred when $\gamma<2$ and the maximum erosion occurred in the ranges $\gamma<0.3$ and $1.2<\gamma<1.4$. Shima et al. [25] and Tomita et al. [26] studied the erosion potential of single bubble collapse in the range $0.14<\gamma<17.1$, and observed three types of bubble collapse modes based on the wall pressure history.

There have been many studies on the relationship between wall pressure and bubblewall dimensionless distance, but there is still a lack of in-depth numerical investigation on the variation of acoustic energy in the flow field during the collapse phase. Some studies [27-29] considered acoustic energy as dissipated energy and derived it from the difference between total energy and other forms of energy (such as kinetic energy, bubble internal energy, bubble potential energy, etc.) from the perspective of energy conservation. In addition, some studies [20,30] obtained acoustic energy from the shock wave energy formula of Cole [31]. For example, Schenke et al. [30] investigated the radiated shock wave energy based on the energy balance approach, however, since the pure vapor and liquid phases in the physical model are considered incompressible, the acoustic pressure perturbation in the shock wave energy formula cannot be directly obtained by simulation results, and needs to be reconstructed by surface specific impact power. Based on weakly compressible potential flow theory, Wang [32] derived a simple formula for the radiated energy associated with acoustic radiation in terms of the bubble volume history using the boundary integral method. However, the research did not take the effect of phase transition into account, which has been proven to have an effect on the bubble rebound radius $[33,34]$, thus inevitably affecting the acoustic radiation energy during the first collapse. Besides, there was also a study [35] considering the phase transition and compressibility, however, the study was limited to the case of bubble spherical collapse, lacking the investigation of bubble non-spherical collapse in the vicinity of the rigid wall.

The open source package OpenFOAM [36] has been widely applied to investigate the bubble collapse behaviors. OpenFOAM (version v1906, https: / www.openfoam.com, 
accessed on 10 December 2021) includes a compressible two-phase solver called compressibleInterFoam to simulate interface problems involving heat transfer. However, the original solver cannot be used to solve the phase-change problems. Therefore, a compressible two-phase model was proposed by modifying the incompressible two-phase model in OpenFOAM. Besides, the compressible two-phase model was further developed to include the equation of state and the energy equation.

The primary aim of this study is to develop a compressible two-phase model accounting for the phase-change to explore the process of acoustic emission, and to investigate the relationship between the acoustic energy and the distance between the bubble and the wall in detail, which lays the foundation for the establishment of subsequent cavitation model. In this paper, we are not concerned with the influence of the wall on the growth stage of the bubble; instead, we only focus on the variation of acoustic energy in the flow field during the bubble collapse phase under the conditions of different $\gamma$-values.

The sections of the present paper are arranged as follows. The governing equations and specific implementation method are shown in Section 2. Section 3 validates the simulation results against the experimental results, and discusses the influence of dimensionless bubble-wall distance on the acoustic energy in the flow field.

\section{Numerical Modeling}

\subsection{Governing Equations}

In the present study, a simplified pure vapor bubble model is used, and its dynamics are considered to be dominated by inertia and compressibility effects. The compressibility and viscosity of the liquid, thermodynamic effects, phase transitions and surface tension of the interface are all taken into account since they may affect the jet formation and the release of acoustic energy during the final stage of the collapse phase [37,38]. In addition, gravity is neglected due to the short existence and small size of the bubble.

Under the above assumptions, the liquid-vapor two-phase flow is treated as a homogeneous mixture applying volume of fluid(VOF) method, hence the continuity and the momentum equations are given as:

$$
\begin{gathered}
\frac{\partial \rho}{\partial t}+\nabla \cdot(\rho \mathbf{U})=0, \\
\frac{\partial(\rho \mathbf{U})}{\partial t}+\nabla \cdot(\rho \mathbf{U U})=-\nabla p+2 \nabla \cdot(\mu \mathbf{D})-\frac{2}{3} \nabla(\mu \nabla \cdot \mathbf{U})+\sigma \kappa \mathbf{n},
\end{gathered}
$$

in which $\nabla$. denotes the divergence, $\nabla$ is the gradient, $\rho$ is the mixture density, $t$ is the time, $\mathbf{U}$ is the mixture velocity, $p$ is the pressure, $\mu$ is the mixture viscosity, $\mathbf{D}=\left[\nabla \mathbf{U}+(\nabla \mathbf{U})^{T}\right] / 2$ is the deformation tensor, $\sigma=0.0725 \mathrm{~N} / \mathrm{m}$ is the surface tension coefficient, $\kappa$ is the mean curvature of the interface, and $\mathbf{n}$ is the unit normal vector of the interface.

The fluid volume fractions $\alpha_{i}$ are introduced to distinguish the vapor bubble $(v)$ and the liquid $(l)$, where $\alpha_{v}=1$ represents vapor phase, $\alpha_{l}=1$ represents liquid phase and $\alpha_{v}+\alpha_{l}=1$ for a binary mixture. Therefore, the mixture density $\rho$ and viscosity $\mu$ are determined by:

$$
\begin{aligned}
& \rho=\alpha_{v} \rho_{v}+\alpha_{l} \rho_{l}, \\
& \mu=\alpha_{v} \mu_{v}+\alpha_{l} \mu_{l},
\end{aligned}
$$

in which the subscripts $v$ and $l$ represent the properties of vapor and liquid, respectively. In the paper, $\rho_{l}=996.558 \mathrm{~kg} / \mathrm{m}^{3}, \rho_{v}=0.017 \mathrm{~kg} / \mathrm{m}^{3}, \mu_{l}=8.538 \times 10^{-4} \mathrm{~Pa} \mathrm{~s}$ and $\mu_{v}=9.919 \times 10^{-6} \mathrm{~Pa}$ s. Besides, the mass equations for both fluids can be written as:

$$
\frac{\partial\left(\alpha_{i} \rho_{i}\right)}{\partial t}+\nabla \cdot\left(\alpha_{i} \rho_{i} \mathbf{U}\right)= \pm \dot{m},(i=v, l)
$$

in which $\dot{m}=\dot{m}^{+}-\dot{m}^{-}$is the net mass transfer rate, $\dot{m}^{+}$is the condensation rate of vapor phase and $\dot{m}^{-}$is the vaporization rate of liquid phase. To account for the effect 
of phase transition process, we introduce the Schnerr-Sauer cavitation model, which has been validated for spherical collapse and aspherical collapse [39]. In the Schnerr-Sauer cavitation model, the condensation rate and vaporization rate are defined as:

$$
\begin{gathered}
\dot{m}^{+}=C_{c} \frac{\rho_{l} \rho_{v}}{\rho} \alpha_{l}\left(1-\alpha_{l}\right) \frac{3}{R_{B}} \sqrt{\frac{2}{3} \frac{p-p_{v}}{\rho_{l}}}, \\
\dot{m}^{-}=C_{v} \frac{\rho_{l} \rho_{v}}{\rho} \alpha_{l}\left(1-\alpha_{l}+\alpha_{N u c}\right) \frac{3}{R_{B}} \sqrt{\frac{2}{3} \frac{p_{v}-p}{\rho_{l}}},
\end{gathered}
$$

where $C_{c}$ is the condensation rate coefficient, $C_{v}$ is the vaporization rate coefficient, $R_{B}$ is the cavitation nuclei radius, $\alpha_{N u c}$ is the nucleation site volume-fraction, $p_{v}$ is the saturation vapor pressure. $R_{B}$ and $\alpha_{N u c}$ are defined as:

$$
\begin{gathered}
R_{B}=\left(\frac{1-\alpha_{l}+\alpha_{N u c}}{\alpha_{l}} \frac{3}{4 \pi} \frac{1}{n}\right)^{1 / 3}, \\
\alpha_{N u c}=\frac{n \frac{4}{3} \pi R_{B}^{3}}{1+n \frac{4}{3} \pi R_{B}^{3}},
\end{gathered}
$$

where $n$ is the number of cavitation nuclei per cubic meter. The saturation vapor pressure $p_{v}$ is expressed by the Antoine equation [40]:

$$
p_{v}=\exp \left(A-\frac{B}{T+C}\right),
$$

where $A, B, C$ are Antoine constants, $T$ is temperature inside the bubble. In the paper, the corresponding values are $C_{c}=1, C_{v}=1, R_{B}=1.0 \times 10^{-6} \mathrm{~m}, n=1.6 \times 10^{13} \mathrm{~m}^{-3}$, $A=23.1964 \mathrm{~Pa}, B=3816.44 \mathrm{~Pa} \mathrm{~K}, C=-46.13 \mathrm{~K}$.

Since the mixture is considered as a compressible medium, the equation of state (EOS) of each phase should be supplied to close the equations of motion. The thermal physics of the vapor bubble, however, is still not fully understood due to the contribution from latent heat. The Rayleigh-Plesset equation [41] is applied to set the pressure and density within the vapor as constant values assuming that the pressure inside the vapor bubble can be kept constant during its collapse.The EOS for the liquid phase is described as:

$$
\rho_{l}=\rho_{l 0}+\frac{1}{c_{l}^{2}} p,
$$

with $\rho_{l 0}$ the initial density of liquid and $c_{l}=1500 \mathrm{~m} / \mathrm{s}$ the speed of sound in liquid.

The energy equation reads [42]:

$$
\begin{aligned}
& \frac{\partial(\rho T)}{\partial t}+\nabla \cdot(\rho T \mathbf{U})+\left(\frac{\alpha_{l}}{C_{p l}}+\frac{\alpha_{v}}{C_{p v}}\right)\left[\frac{\partial(\rho K)}{\partial t}+\nabla \cdot(\rho K \mathbf{U})\right] \\
& =\left(\frac{\alpha_{l}}{C_{p l}}+\frac{\alpha_{v}}{C_{p v}}\right)\left[\frac{\partial p}{\partial t}+\nabla \cdot(\boldsymbol{\tau} \cdot \mathbf{U})\right]+\left(\frac{\alpha_{l} \lambda_{l}}{C_{p l}}+\frac{\alpha_{v} \lambda_{v}}{C_{p v}}\right)\left(\nabla^{2} T\right),
\end{aligned}
$$

where $K$ is the kinematic energy, $\tau$ is the shear stress, $C_{p}, \lambda$ are the heat capacity and the thermal conductivity, the subscripts $l$ and $v$ represent liquid and vapor, respectively. In the paper, $C_{p l}=4181.097 \mathrm{~J} /(\mathrm{kg} \mathrm{K}), C_{p v}=1913.953 \mathrm{~J} /(\mathrm{kg} \mathrm{K}), \lambda_{l}=0.677 \mathrm{~W} /(\mathrm{m} \mathrm{K})$ and $\lambda_{v}=0.020 \mathrm{~W} /(\mathrm{m} \mathrm{K})$.

In the current work, the energy discussed includes the bubble potential energy, and the acoustic energy during the bubble collapse. The potential energy is defined as [21]:

$$
E_{\text {pot }}=\frac{4}{3} \pi R^{3} \Delta p,
$$


with $R$ the bubble radius and $\Delta p$ the pressure difference on both sides of the bubble interface. The acoustic energy can be written as the following integral form [43]:

$$
E_{\text {wave }}=\int \frac{\Delta p^{2}}{\left(\rho c_{l}\right)^{2}} d V .
$$

\subsection{Numerical Implementation}

In the present study, the finite volume method is applied to implement the discretization of the formulas since it is more apt to deal with compressible fluids compared with the boundary integral method, the VOF method is utilized to capture the interfaces between two phases. Therefore, on the basis of the assumptions in the previous subsection, a solver for two compressible immiscible fluids with phase transition named compressibleInterPhaseChangeFoam has been developed based on two solvers on OpenFOAM-v1906, interPhaseChangeFoam and compressibleInterFoam.

The simulations are assumed to be axisymmetric to reduce computational cost, the wedge geometry $\left(102 R_{0} \times 104 R_{0}\right)$ with an opening angle set to be $5^{\circ}$ is used in which $R_{0}$ is the bubble initial radius. The bubble center is initiated at a distance $L$ above the rigid wall, as shown in Figure 1a. The mesh is generated with the local refinement using the OpenFOAM utility blockMesh, as shown in Figure $1 \mathrm{~b}$. The refinement region ranges from 0 to $2 R_{0}$ and from 0 to $4 R_{0}$ in $\mathrm{x}$ and $\mathrm{z}$ directions, respectively. In the local refinement zone, the grid spacing is uniform, and the total grid numbers depend on the cell numbers in the radial direction inside the bubble. Outside the refinement zone, however, the grid spacing increases with a progression factor of 1.05 until the boundary of the computational domain.

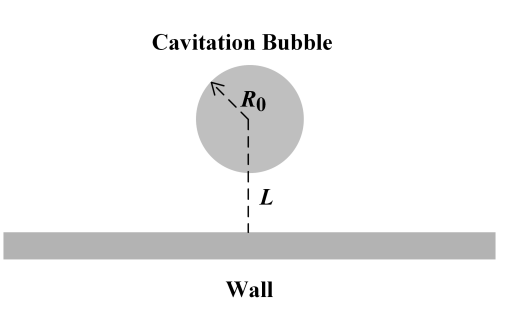

(a)

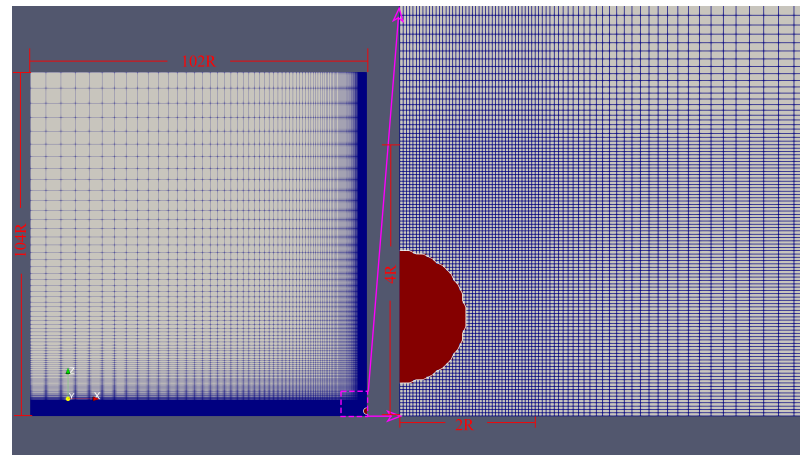

(b)

Figure 1. (a) Schematic showing the configuration of a cavitation bubble and a solid wall, (b) 2D illustration of the domain with local grid refinement for $\gamma=1.5$.

Second-order Euler implicit differencing is applied to the time derivatives. The spatial differencing of the convective terms uses the flux-difference splitting scheme based on van Leer's Monotone Upstream-centered Schemes for Conservation Laws(MUSCL) method. A second-order central differencing is used for the viscous terms. The pressure-velocity coupling is handled via the Pressure-Implicit with Splitting of Operators(PISO) algorithm. The time-step is set small enough such that the maximum Courant number of the flow is 0.2. Boundary conditions at the wall are no-slip for the velocity and zero normal gradient both for the pressure and the liquid volume fraction $\alpha_{l}$. At the boundary of the computational domain, which is located far from the bubble, the pressure satisfies a fixedValue boundary condition.

\section{Validation and Results}

\subsection{Validation}

To validate the numerical model, the time history of the bubble radius $R_{B}$ in a free flow field is compared with the analytical solution of a spherical bubble in the simplified 
Rayleigh-Plesset equation firstly. Here, we approximate that the vapor bubble collapses spherically in a free flow field when $\gamma=50$. The flow field is static at initial time, and the initial radius of the vapor bubble $R_{0}=2 \mathrm{~mm}$. The pressure field inside the bubble, $P_{v}$, and in the liquid, $P_{\infty}$, are initialized as $3540 \mathrm{~Pa}$ and $101,325 \mathrm{~Pa}$, respectively. A fixed pressure value of $P_{\infty}=101,325 \mathrm{~Pa}$ is imposed at the far field boundaries.

Results are shown in a dimensionless form for better comparison. The bubble radius $R_{B}$ and time $t$ are nondimensionalized as:

$$
\begin{gathered}
R^{*}=R_{B} / R_{0} \\
t^{*}=t / t_{c},
\end{gathered}
$$

where $t_{c}$ is the time span obtained from the analytical solution of the evolution of a spherical bubble for the simplified Rayleigh-Plesset equation [41], with the formula

$$
t_{c} \approx 0.915 R_{0} \sqrt{\frac{\rho_{l}}{p_{\infty}-p_{v}}} .
$$

In addition, five different mesh resolutions, that is, 45, 100, 150, 200, 250 cells in the radial direction inside the bubble, are set to inspect the spatial resolution requirement in the numerical simulation. Figure 2 shows the numerical simulation results of the above five resolutions, compared with the analytical solution. It is found that for the five cases with different resolutions, the evolution of the bubble radius in the first and middle stages of the collapse is consistent with the analytical solution. In the final stage of the collapse, however, a significant time delay is observed between the numerical results and the analytical solution. The key reason is that the simplified Rayleigh-Plesset equation does not take the phase transition and heat transfer effect in the numerical model into account, which can exert a retardation effect on the collapse of the bubble. Besides, for grid independent studies, the time delay magnitude decreases with the cell numbers when the cell numbers in the radial direction inside the bubble ranges from 45 to 150 , while the time delay magnitude basically does not vary with cell numbers when the cell numbers in the radial direction inside the bubble is larger than 150 . Therefore, we select a case with 150 cells in the radial direction inside the bubble for further validation and simulation.

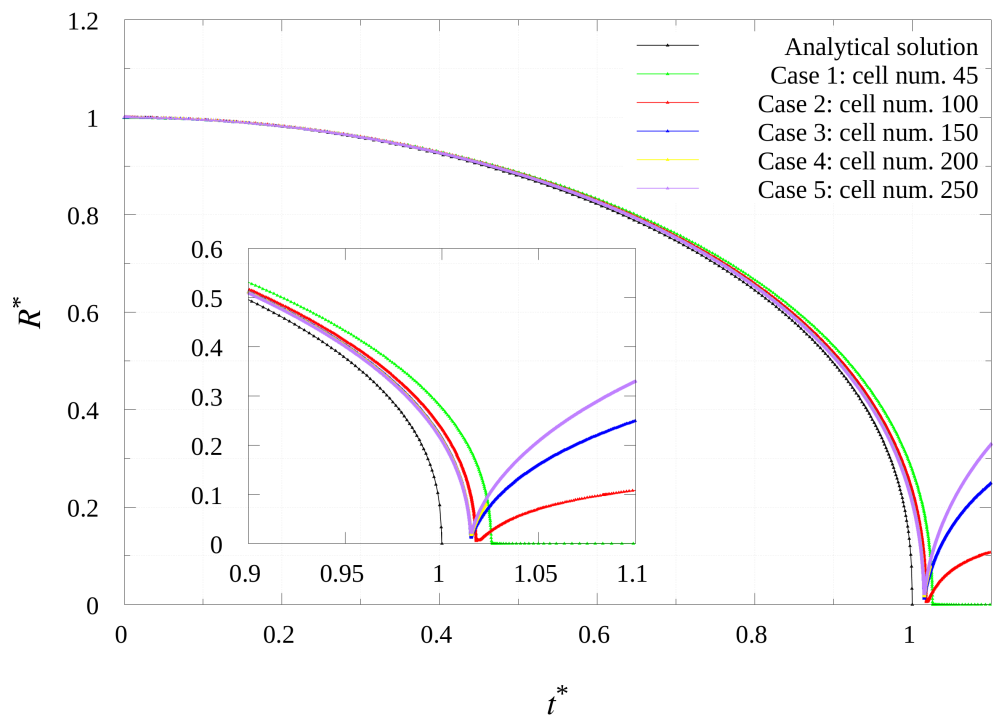

Figure 2. Comparisons of temporal evolution of bubble radius between analytical solution and numerical results with different cells in the bubble.

As can be seen from Formula (14), the acoustic energy is closely related to the pressure field, and thus the pressure field needs to be further verified to ensure the accuracy of acoustic energy. Supponen et al. [44] established a model to measure shock wave peak 
pressures for bubbles deformed by gravity, a rigid and a free surface and obtained the relationship between peak pressures and various parameters, for example, $R_{0}, c_{l}, \rho$, and so forth, as shown below:

$$
P_{\max } \propto\left(\rho c_{l}^{2} \Delta p\right)^{1 / 2}\left(\frac{R_{0}}{d}\right)^{\beta} \zeta^{(2 \beta / 3-1)},
$$

in which $P_{\max }$ is the peak pressure measured, $d$ is the distance between the bubble center and points to be measured, $\beta$ is a free parameter typically about 2 in the near field and 1.1 in the far field of the bubble center, $\zeta \equiv|\zeta|, \zeta$ is a dimensionless vector-parameter related to $\gamma$, expressed as the following formula under the condition of the flat rigid surface,

$$
\zeta=-0.195 \gamma^{-2} \mathbf{n}
$$

where $\mathbf{n}$ is the unit vector normal to the surface, directed from the surface to the bubble. Figure 3 displays the numerical simulation results of the peak pressures, $\beta=2$ is set here as the points selected are in the near field of the bubble center. Black dots identify the peak pressures of different points in the cases with different $\gamma$-values. The red line indicates curve fitting of peak pressures. It can be seen that the peak pressures are proportional to the parameters mentioned above, which shows a good agreement with the empirical correlation proposed in the literature.

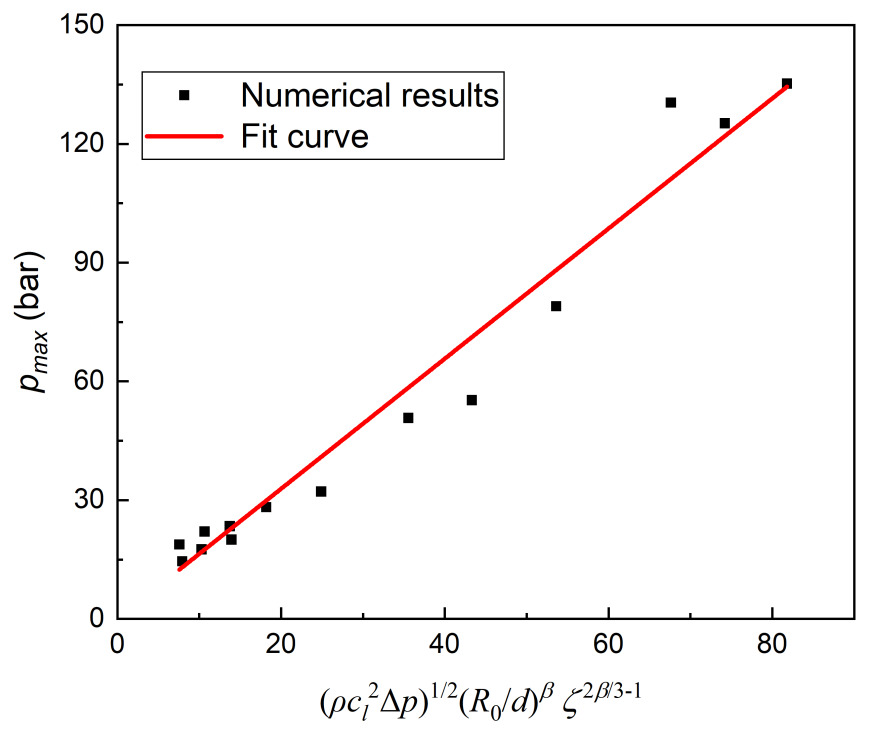

Figure 3. The maximum pressure of numerical results in the cases with different $\gamma$-values.

\subsection{Results}

Figure 4 shows a time series of the collapse of the vapor bubble with $\gamma=1.1$. Initially, the ambient pressure is much higher than the pressure inside the vapor bubble, thus inertia forces are predominant, and the bubble starts to collapse (Figure 4a). The pressure gradient at the bottom of the bubble is less than that at the top of the bubble in the later stage of collapse due to the existence of the rigid wall, so that the bottom of the bubble collapses slower, leading to the formation of an oval-shaped bubble (Figure $4 \mathrm{~b}$ ). Meanwhile, a jet toward the rigid wall is generated at the top of the bubble. Subsequently, with the continuous impact of the jet on the bubble surface, the bubble gradually assumes a "heartshape". In addition, during the jet impingement process, the pressure gradient above the bubble top increases continuously, which leads to the further acceleration of the jet and aggravates the bubble collapse process (Figure 4c). In the final stage of collapse, the jet completely penetrates the lower surface of the bubble and continues to impact the rigid wall. At the same time, the bubble becomes a toroidal bubble and a high pressure region 
is generated below the bubble, indicating the first release of acoustic energy produced by the jet penetrating the bubble (Figure $4 \mathrm{~d}$ ). Finally, when the bubble reaches its minimum volume, the pressure difference between the inside and outside of the bubble reaches its maximum value, allowing a second stronger spherical shock wave to be emitted within a very short time (Figure 4e). Next in the rebound process of the bubble, the shock wave propagates radially outwards, hits the rigid wall and is reflected (Figure $4 \mathrm{f}$ ).

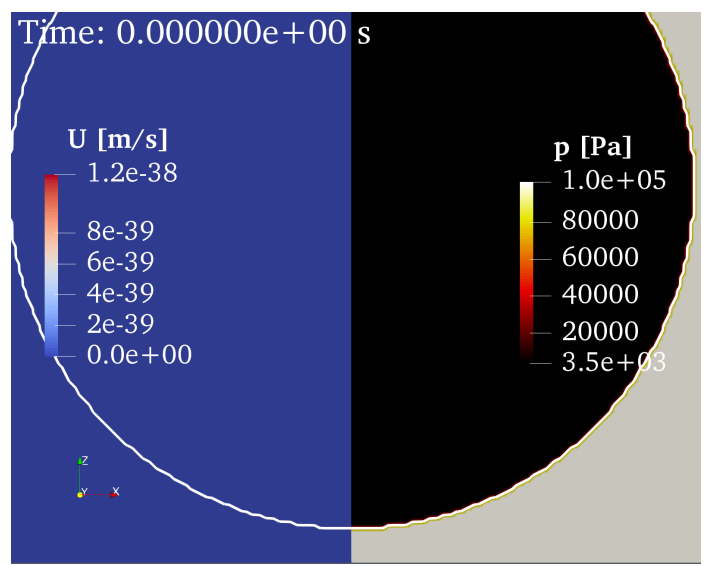

(a)

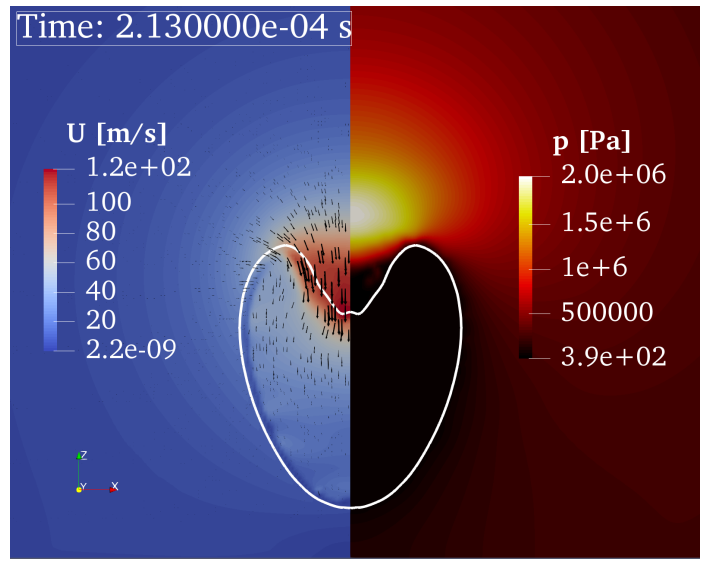

(c)

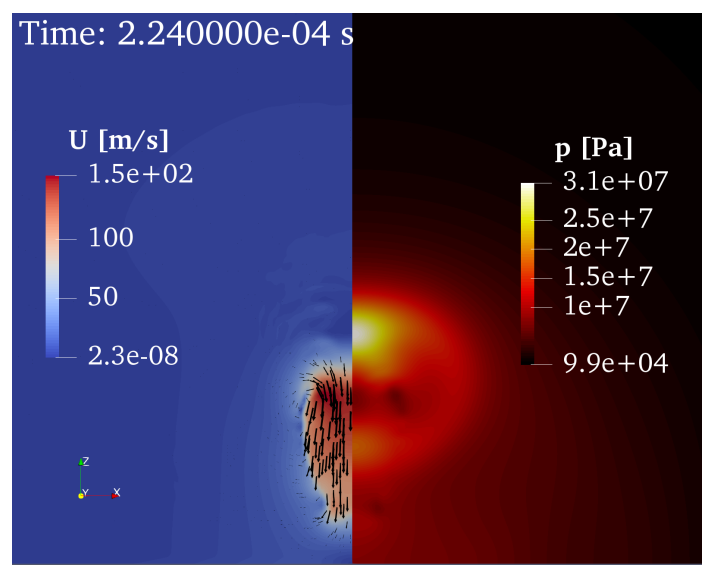

(e)

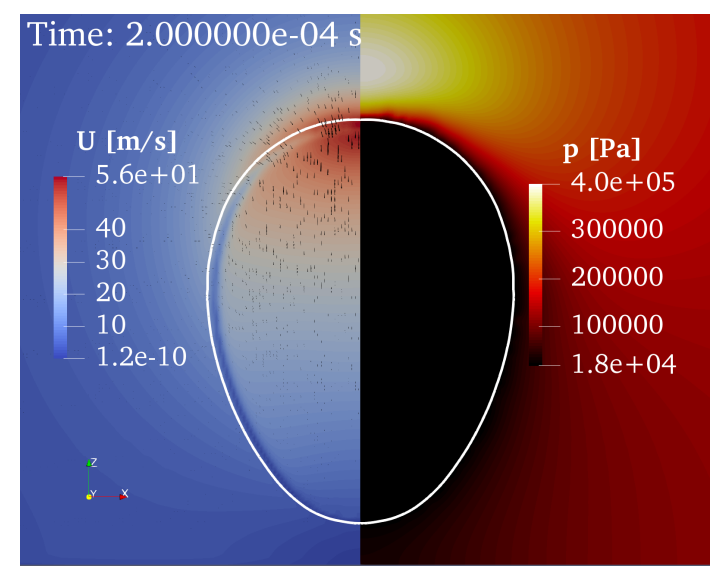

(b)

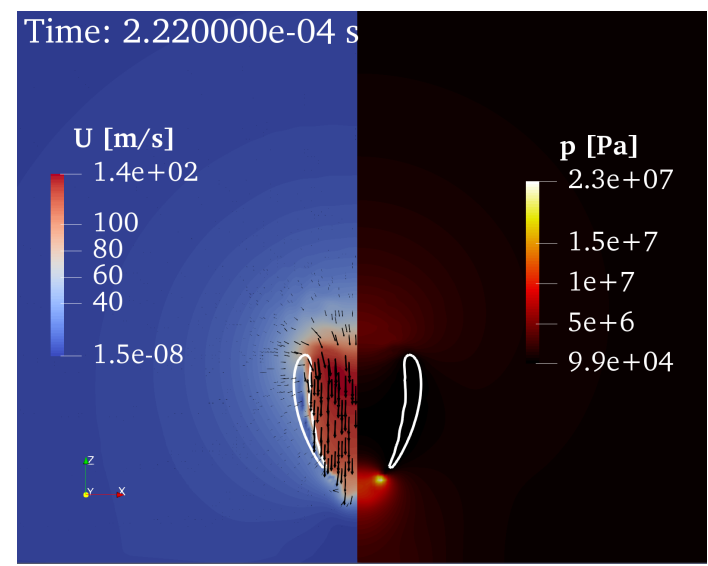

(d)

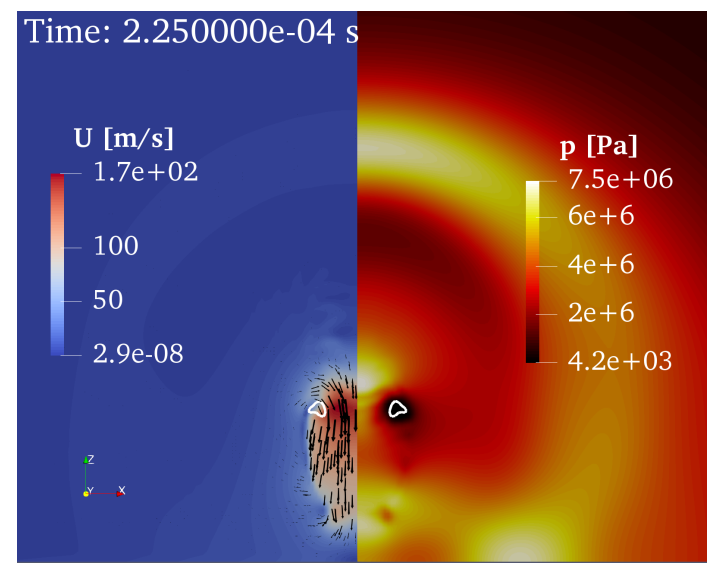

(f)

Figure 4. Velocity and pressure fields for temporal evolution of the bubble at $\gamma=1.1$. (a) $t^{*}=0$, (b) $t^{*}=1.083,(\mathbf{c}) t^{*}=1.151,(\mathbf{d}) t^{*}=1.202,(\mathbf{e}) t^{*}=1.213$, and (f) $t^{*}=1.219$. 
Figure 5 plots the temporal evolution of the acoustic energy, obtained from the numerical simulations with different $\gamma$-values. The interest region ranges from $\gamma=0.1$ to 2.0. The acoustic energy is normalized as:

$$
E_{\text {wave }}^{*}=\frac{E_{\text {wave }}}{E_{\text {pot0 }}},
$$

where $E_{\text {pot } 0}$ is the initial bubble potential energy. In Figure 5, we observe that for the cases with different $\gamma$-values, the acoustic energy is always at a low level during the whole bubble collapse process, and rises to a high level within a very short time after the first bubble collapse. It has been shown analytically that [28,32], the main forms of energy in the flow field are bubble potential energy and fluid kinetic energy in most of the time of the collapse process, while the acoustic energy and bubble internal energy are at a low level. At the end of the collapse process, the internal energy of the bubble rises significantly and is partially converted into acoustic energy after the collapse, thus resulting in a rapid rise in acoustic energy. In addition, we also note that the rising time and amplitude of acoustic energy are related to the $\gamma$-values. Therefore, the relationship between $\gamma$ and maximum acoustic energy, normalized time of maximum acoustic energy, and bubble collapse time is further compared in Figure 6. Among them, we can clearly see that the results of the case with $\gamma=0.9$ are critical. The maximum acoustic energy increases with $\gamma$-values for $\gamma>0.9$ but decreases with $\gamma$-values for $\gamma<0.9$, which is in good agreement with the experimental results in the literature [45]. The variation trends of normalized time of maximum acoustic energy and bubble collapse time, however, are opposite to that of maximum acoustic energy. For large $\gamma$-value, when $\gamma$-value increases, the relative distance between the bubble center and the rigid wall becomes farther, the influence of the wall surface on the bubble collapse process decreases, the jet strength is weaker, and water is more inclined to flow radially from the surrounding region of the bubble to the bubble center, which results in the more violent bubble collapse, and thus leads to stronger acoustic energy release after the bubble collapse. For the case of $\gamma$-value close to 0.9 , a vortex ring gradually moving towards the wall is formed around the bubble after the jet penetrates the lower surface of the bubble(Figure 7a), which reduces the compressibility of the bubble and alleviates the subsequent acoustic release compared with the case of large $\gamma$-value. While for small $\gamma$-value, when $\gamma$-values decreases, the bubble is closer to hemispherical shape, and the behavior of the bubble in the collapse process is more similar to that of the bubble in the far-field. Taking the case of $\gamma=0.3$ as an example(Figure $7 \mathrm{~b}$ ), the strength of the vortex ring is reduced compared to the case of $\gamma=0.9$, causing a stronger compression of the bubble and acoustic emission.

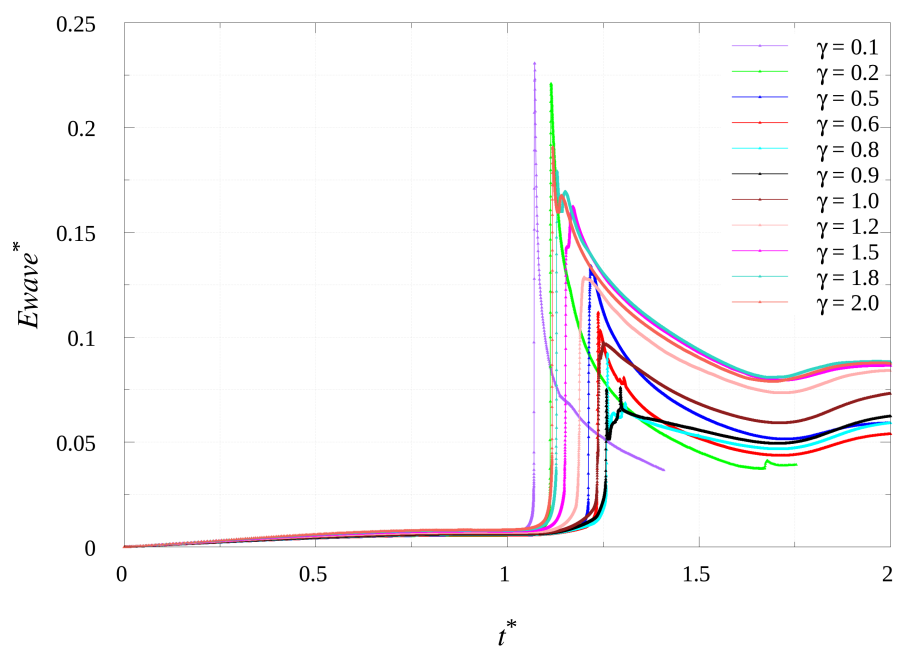

Figure 5. Temporal evolution of the acoustic energy in the field with different $\gamma$-values. 


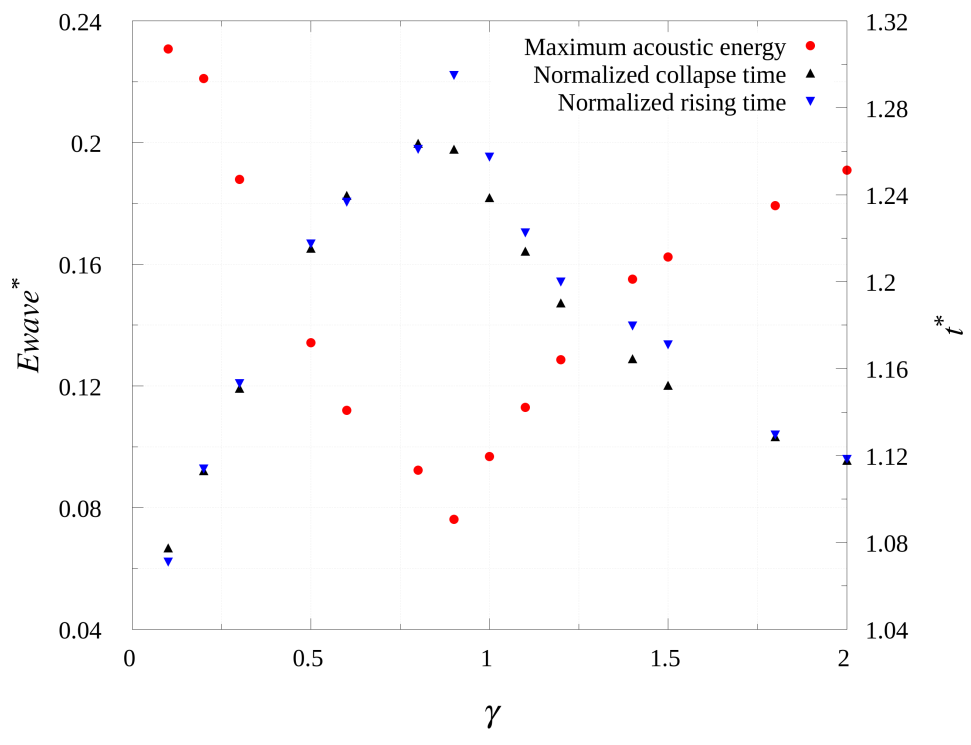

Figure 6. Diagram of the relationship between the maximum acoustic energy, normalized time of maximum acoustic energy, bubble collapse time and $\gamma$.

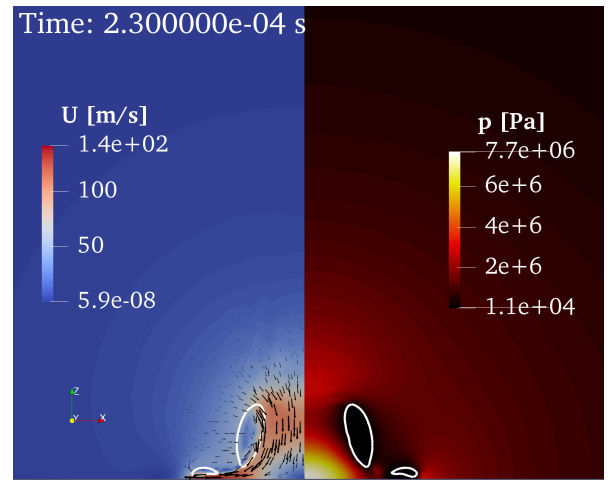

(a)

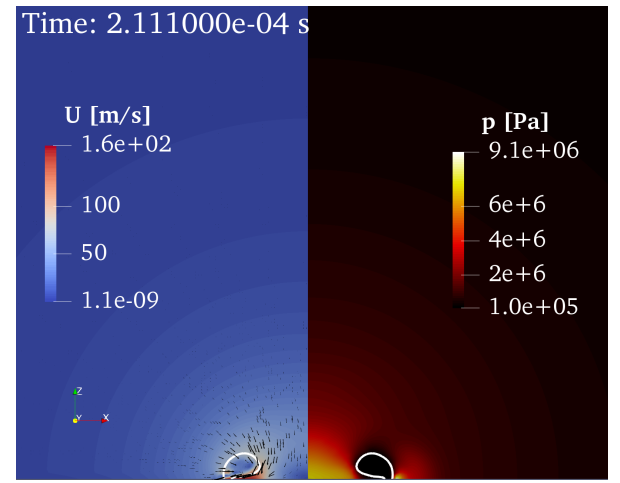

(b)

Figure 7. Comparison diagram of velocity and pressure fields with different $\gamma$-values. (a) $\gamma=0.9$, (b) $\gamma=0.3$.

\section{Conclusions}

In the present study, we developed a compressible two-phase solver on OpenFOAMv1906 to numerically investigate the cavitation bubble dynamic in the vicinity of a rigid wall. To obtain the accuracy pressure field directly, we take the compressibility and phase transition into account. Meanwhile, the grid independence analysis was performed to reduce the computational cost and improve the accuracy of simulation results. For this purpose, we conducted simulations of the collapse of a vapor bubble in a free flow field with different cell numbers in the radial direction inside the bubble. By comparing the numerical results of the evolution of the bubble radius with the analytical solution of the evolution of a spherical bubble for the simplified Rayleigh-Plesset equation, we eventually selected the case with 150 cells in the radial direction inside the bubble for further study. Subsequently, we verified the accuracy of the pressure field during the bubble collapse near a rigid wall. The simulation results showed a good agreement with the empirical correlation proposed in the literature.

Furthermore, we discussed the emission process of acoustic energy during the bubble collapse at $\gamma=1.1$. The first release of acoustic energy was produced by the jet penetrating the bubble, and the second stronger spherical shock wave was emitted within a very short time after the bubble reaches its minimum volume. Besides, we investigated the temporal evolution of the acoustic radiation energy in the flow field with different $\gamma$-values. For 
different $\gamma$-values, the time-varying curves of acoustic energy have similar shapes. The acoustic energy is at a low level during the most of the time of the collapse process, and rises significantly after the bubble reaches its minimum volume. However, the effect of $\gamma$ on the amplitude and the rising time of acoustic energy is obvious. The amplitude of acoustic energy decreases with $\gamma$ in the range $0.1<\gamma<0.9$ while increases with $\gamma$ in the range $0.9<\gamma<2$.0. For cases of $\gamma>0.9$, the influence of the wall on the bubble collapse process decreases with $\gamma$, the jet strength is weaker, thus leads to more violent bubble collapse and stronger acoustic energy. For cases of $\gamma<0.9$, the strength of the vortex ring is reduced with $\gamma$, causing a stronger compression of the bubble and acoustic emission.

Author Contributions: Conceptualization, L.C.; methodology, L.C.; software, L.C. and H.D.; validation, H.D.; formal analysis, L.C. and H.D.; investigation, L.C. and H.D.; resources, X.L.; data curation, H.D.; writing—original draft preparation, H.D.; writing—review and editing, L.C.; visualization, H.D.; funding acquisition, X.L. All authors have read and agreed to the published version of the manuscript.

Funding: This research was funded by National Natural Science Foundation of China, grant number 51871206; the National Key Research and Development Programmes of China, grant number 2017YFF0210704; and Zhejiang Provincial Natural Science Foundation, grant number LQ20E090005.

Institutional Review Board Statement: Not applicable.

Informed Consent Statement: Not applicable.

Data Availability Statement: Not applicable.

Conflicts of Interest: The authors declare no conflict of interest.

\section{References}

1. Gohil, P.P.; Saini, R. Coalesced effect of cavitation and silt erosion in hydro turbines-A review. Renew. Sustain. Energy Rev. 2014, 33, 280-289. [CrossRef]

2. Arndt, R.E. Some remarks on hydrofoil cavitation. J. Hydrodyn. Ser. B 2012, 24, 305-314. [CrossRef]

3. Escaler, X.; Egusquiza, E.; Farhat, M.; Avellan, F.; Coussirat, M. Detection of cavitation in hydraulic turbines. Mech. Syst. Signal Process. 2006, 20, 983-1007. [CrossRef]

4. Avellan, F. Introduction to Cavitation in Hydraulic Machinery; Technical Report; Politehnica University of Timișoara: Timișoara, Romania, 2004.

5. Dorji, U.; Ghomashchi, R. Hydro turbine failure mechanisms: An overview. Eng. Fail. Anal. 2014, 44, 136-147. [CrossRef]

6. Neopane, H.P.; Cervantes, M. Sediment erosion in hydraulic turbines. Glob. J. Res. Eng. 2011, 11, 16-26.

7. Brennen, C.E. Cavitation and Bubble Dynamics; Cambridge University Press: Cambridge, UK, 2014.

8. Franc, J.P.; Michel, J.M. Fundamentals of Cavitation; Springer Science \& Business Media: Berlin/Heidelberg, Germany, 2006; Volume 76.

9. Kornfeld, M.; Suvorov, L. On the destructive action of cavitation. J. Appl. Phys. 1944, 15, 495-506. [CrossRef]

10. van Rijsbergen, M.; Foeth, E.J.; Fitzsimmons, P.; Boorsma, A. High-speed video observations and acoustic-impact measurements on a NACA 0015 foil. In Proceedings of the 8th International Symposium on Cavitation, Singapore, 13-16 August 2012; pp. 958-964.

11. Pfitsch, W.; Gowing, S.; Fry, D.; Donnelly, M.; Jessup, S. Development of measurement techniques for studying propeller erosion damage in severe wake fields. In Proceedings of the 7th International Symposium on Cavitation, Ann Arbor, MI, USA, 16-20 August 2009.

12. Cao, Y.; Peng, X.; Yan, K.; Xu, L.; Shu, L. A qualitative study on the relationship between cavitation structure and erosion region around a $3 \mathrm{~d}$ twisted hydrofoil by painting method. In Proceedings of the Fifth International Symposium on Marine Propulsors, Espoo, Finland, 12-15 June 2017.

13. Arabnejad, M.H.; Svennberg, U.; Bensow, R.E. Numerical assessment of cavitation erosion risk using incompressible simulation of cavitating flows. Wear 2021, 464, 203529. [CrossRef]

14. Berchiche, N.; Grekula, M.; Bark, G. Concept of Focusing of Collapse Energy-Application to Cavitation Observations. In Proceedings of the 5th International Symposium on Cavitation (CAV 2003), Osaka, Japan, 1-4 November 2003; pp. 1-4.

15. Dular, M.; Coutier-Delgosha, O. Numerical modelling of cavitation erosion. Int. J. Numer. Methods Fluids 2009, 61, 1388-1410. [CrossRef]

16. Peters, A.; Sagar, H.; Lantermann, U.; el Moctar, O. Numerical modelling and prediction of cavitation erosion. Wear 2015, 338, 189-201. [CrossRef] 
17. Peters, A.; Lantermann, U.; el Moctar, O. Numerical prediction of cavitation erosion on a ship propeller in model-and full-scale. Wear 2018, 408-409, 1-12. [CrossRef]

18. Patella, R.F.; Reboud, J.L.; Briançon-Marjollet, L. A phenomenological and numerical model for scaling the flow agressiveness in cavitation erosion. In Cavitation Erosion Workshop; Val de Reuil, France, 2004. Available online: https://slideplayer.com/amp/12 $767153 /$ (accessed on 10 December 2021).

19. Patella, R.F.; Archer, A.; Flageul, C. Numerical and experimental investigations on cavitation erosion. In IOP Conference Series: Earth and Environmental Science; IOP Publishing: Bristol, UK, 2012; Volume 15, p. 022013.

20. Fortes-Patella, R.; Challier, G.; Reboud, J.L.; Archer, A. Energy balance in cavitation erosion: From bubble collapse to indentation of material surface. J. Fluids Eng. 2013, 135, 011303. [CrossRef]

21. Hammitt, F.G. Observations on cavitation damage in a flowing system. J. Basic Eng. 1963, 85, 347-356. [CrossRef]

22. Schenke, S.; van Terwisga, T.J. An energy conservative method to predict the erosive aggressiveness of collapsing cavitating structures and cavitating flows from numerical simulations. Int. J. Multiph. Flow 2019, 111, 200-218. [CrossRef]

23. Melissaris, T.; Bulten, N.; van Terwisga, T.J. On the applicability of cavitation erosion risk models with a URANS solver. J. Fluids Eng. 2019, 141, 101104. [CrossRef]

24. Philipp, A.; Lauterborn, W. Cavitation erosion by single laser-produced bubbles. J. Fluid Mech. 1998, 361, 75-116. [CrossRef]

25. Shima, A.; Takayama, K.; Tomita, Y.; Ohsawa, N. Mechanism of impact pressure generation from spark-generated bubble collapse near a wall. Aiaa J. 1983, 21, 55-59. [CrossRef]

26. Tomita, Y.; Shima, A. Mechanisms of impulsive pressure generation and damage pit formation by bubble collapse. J. Fluid Mech. 1986, 169, 535-564. [CrossRef]

27. Vokurka, K. Significant intervals of energy transforms in bubbles freely oscillating in liquids. J. Hydrodyn. 2017, 29, 217-225.

28. Tian, Z.L.; Liu, Y.L.; Zhang, A.M.; Tao, L. Energy dissipation of pulsating bubbles in compressible fluids using the Eulerian finite-element method. Ocean Eng. 2010, 196, 106714. [CrossRef]

29. Tinguely, M.; Obreschkow, D.; Kobel, P.; Dorsaz, N.; De Bosset, A.; Farhat, M. Energy partition at the collapse of spherical cavitation bubbles. Phys. Rev. E 2012, 86, 046315. [CrossRef]

30. Schenke, S.; Melissaris, T.; Van Terwisga, T. On the relevance of kinematics for cavitation implosion loads. Phys. Fluids 2019, 31, 052102. [CrossRef]

31. Cole, R. Underwater Explosions; Princeton University Press: Princeton, NJ, USA, 1948.

32. Wang, Q. Local energy of a bubble system and its loss due to acoustic radiation. J. Fluid Mech. 2016, 797, 201-230. [CrossRef]

33. Christian, C.M. Modeling Laser-Generated Cavitation Bubbles. Master's Thesis, Pennsylvania State University, College of Engineering, University Park, PA, USA, 2012.

34. Zeng, Q.; Gonzalez-Avila, S.R.; Dijkink, R.; Koukouvinis, P.; Gavaises, M.; Ohl, C.D. Wall shear stress from jetting cavitation bubbles. J. Fluid Mech. 2018, 846, 341-355. [CrossRef]

35. Aganin, A.; Ganiev, O.; Davletshin, A.; Ukrainskii, L. Evaluation of Thermal and Acoustic Energy during Collapse of Cavitation Bubbles. J. Mach. Manuf. Reliab. 2020, 49, 367-373. [CrossRef]

36. Weller, H.G.; Tabor, G.; Jasak, H.; Fureby, C. A tensorial approach to computational continuum mechanics using object-oriented techniques. Comput. Phys. 1998, 12, 620-631. [CrossRef]

37. Koch, M.; Lechner, C.; Reuter, F.; Köhler, K.; Mettin, R.; Lauterborn, W. Numerical modeling of laser generated cavitation bubbles with the finite volume and volume of fluid method, using OpenFOAM. Comput. Fluids 2016, 126, 71-90. [CrossRef]

38. Lechner, C.; Koch, M.; Lauterborn, W.; Mettin, R. Pressure and tension waves from bubble collapse near a solid boundary: A numerical approach. J. Acoust. Soc. Am. 2017, 142, 3649-3659. [CrossRef] [PubMed]

39. Schnerr, G.H.; Sezal, I.H.; Schmidt, S.J. Numerical investigation of three-dimensional cloud cavitation with special emphasis on collapse induced shock dynamics. Phys. Fluids 2008, 20, 040703. [CrossRef]

40. Antoine, C. Tension des vapeurs: Nouvelle relation entre les tension et les temperatures. Comptes Rendus 1888, 107, 681-684.

41. Rayleigh, L. On the pressure developed in a liquid during the collapse of a spherical cavity. Phil. Mag. 1917, 34, 94-98. [CrossRef]

42. Yin, J.; Zhang, Y.; Zhu, J.; Lv, L.; Tian, L. An experimental and numerical study on the dynamical behaviors of the rebound cavitation bubble near the solid wall. Int. J. Heat Mass Transf. 2021, 177, 121525. [CrossRef]

43. Zhang, J.; Zhang, L.; Deng, J. Numerical study of the collapse of multiple bubbles and the energy conversion during bubble collapse. Water 2019, 11, 247. [CrossRef]

44. Supponen, O.; Obreschkow, D.; Kobel, P.; Tinguely, M.; Dorsaz, N.; Farhat, M. Shock waves from nonspherical cavitation bubbles. Phys. Rev. Fluids 2017, 2, 093601. [CrossRef]

45. Vogel, A.; Lauterborn, W. Acoustic transient generation by laser-produced cavitation bubbles near solid boundaries. J. Acoust. Soc. Am. 1988, 84, 719-731. [CrossRef] 